\title{
Neural Implications of COVID-19 on Patients, Healthcare Workers, and the General Population
}

\author{
Mona Reddy Kurra1, Bryant Chung ${ }^{1}$, Akrom Ali, Arianne Vethan, Arohee Agarwal, \\ Casey Syal ${ }^{1}$, Crystal Ma ${ }^{1}$, Kathlyn Nicole Periquet ${ }^{1}$, Madison Park ${ }^{1}$, Noah Park ${ }^{1}$, Sana \\ Asifriyaz ${ }^{1}$, Teana Gomez ${ }^{1}$, Vinutha Sandadi ${ }^{1}$, Dr. Paul Matthews ${ }^{2}$, and Dr. Matt Wright ${ }^{2}$ \\ ${ }^{1}$ Oxford Academy, Cypress, CA, USA \\ ${ }^{2}$ The Lundquist Institute, Torrance, CA, USA
}

\section{ABSTRACT}

There is evidence that coronavirus 2019 (COVID-19) has significant direct and indirect effects on the brain, including neuronal degeneration and disturbed consciousness. Thus, it is important to examine the neural implications of the novel virus to address long term issues regarding brain functioning and mental health. This review first focuses on how COVID-19 might, and previous coronaviruses do directly infiltrate the brain. Next, we discuss how COVID-19 indirectly affects the mental health of nurses and the public resulting from work pressures and paranoia over contracting the virus. Finally, we describe the variable response to the pandemic related to cultural factors, the impact of leadership style to the pandemic response, and the effects of COVID-19 on people with lower socioeconomic status.

\section{History}

Coronaviruses in the past have infiltrated parts of the nervous system and are now being compared to the current COVID-19 pandemic caused by severe acute respiratory syndrome coronavirus 2 (SARS-CoV-2).

\section{SARS-CoV}

Severe acute respiratory syndrome started in Asia and spread throughout the world in 2003. It causes neurological injury such as polyneuropathy, encephalitis, and aortic ischemic stroke (Tsai et al., 2005). Autopsies performed on severe acute respiratory syndrome (SARS) victims revealed signs of cerebral edema and meningeal vasodilation in most cases of SARS, as well as infiltration of monocytes and lymphocytes in the vessel wall, ischemic changes of neurons, and demyelination of nerve fibers. SARS-CoV virus particles and genome sequences were also seen in the brain (Gu et al., 2005, Zhang et al., 2003).

\section{MERS-CoV}

Middle East respiratory syndrome spread in 2012 and was localized to the Arabian Peninsula. It originated from bats and was mostly transferred to humans through infected camels. Middle East respiratory syndrome (MERS-CoV) is potentially neuroinvasive, as a study identified that $25.7 \%$ of patients developed confusion and that $8.6 \%$ of patients had seizures (Saad et al., 2014). Additionally, $1 / 5$ of patients showed neurological symptoms. Some of these included a disturbance of consciousness, paralysis that may be attributed to Guillain-Barre syndrome, and ischemic stroke (Kim et al., 2017). Interestingly, these neurological complications occurred 2-3 weeks after respiratory symptoms such as fever and cough. (Kim et al., 2017). 


\section{SARS-CoV-2}

SARS-CoV-2 shares approximately $79.5 \%$ genetic similarity with SARS-CoV and about $50 \%$ similarity with MERSCoV (Wu et al., 2020, Lu et al., 2020, Wang et al., 2020). Clinical data revealed symptoms similar to intracranial infections such as headache, epilepsy, and disturbed consciousness. Additionally, anosmia and dysgeusia was found in COVID-19 patients (Chiu et al., 2020, Giacomelli et al., 2020, Cho et al., 2020, Hopkins et al., 2020). Unlike MERS-CoV, some patients developed COVID-19 related symptoms such as fever only after neurologic symptoms (Mao et al., 2020). In fact, researchers were able to use genome sequencing to confirm the presence of SARS-CoV-2 in cerebrospinal fluid, further demonstrating that the virus can infiltrate the nervous system (Xiang et al., 2020).

\section{Paths}

COVID-19 and previous coronaviruses have been able to infiltrate the brain through multiple pathways:

\section{Olfactory Bulb}

The organization of olfactory nerves and the olfactory bulb in the nasal cavity and forebrain creates a channel between the nasal epithelium and the central nervous system (CNS). As a result, coronaviruses (CoV) can enter the brain in the early stages of infection. $\mathrm{CoV}$ can then spread through the brain and cerebrospinal fluid in a span of 7 days and cause inflammation and demyelination. (Wu et al., 2020). Additionally, a study examining a patient's olfactory bulb volumes taken three years before COVID-19 induced anosmia found significant decreases in size $\left(49.5 \mathrm{~mm}^{\wedge} 3\right.$ and $47.46 \mathrm{~mm}^{\wedge} 3$ to $29.96 \mathrm{~mm}^{\wedge} 3$ and $35.51 \mathrm{~mm}^{\wedge} 3$, respectively). There was an observed correlation between olfactory bulb volume loss and the duration of olfactory loss, and it was hypothesized that anosmia and dysgeusia in CoV may be caused by olfactory epithelial support cells such as the sustentacular cells and Bowman's gland cells (Chiu et al., 2020). However, it is important to note that the patient olfactory formal assessment (Chiu et al., 2020, Chung et al., 2018). However, there is no literature testing the correlation between olfactory bulb atrophy and olfaction in COVID-19 patients.

\section{Inflammatory Response}

$\mathrm{CoV}$ infections are persistent and are able to infect macrophages, microglia, and astrocytes in the CNS. Neurotropic viruses can activate glial cells to induce a pro-inflammatory state, such as interleukin (IL)-6, an important part of the cytokine storm. IL is linked to the severity of COVID-19 symptoms (Li et al., 2004, Wan et al., 2020). Experiments revealed that primary glial cells cultured in vitro released large quantities of inflammatory factors like IL-6, IL-12, IL-15, and TNF- $\alpha$ (tumor necrosis factor-alpha) after infection from CoV (Bohmwald et al., 2018). These activated immune cells in the brain caused chronic inflammation and brain damage.

\section{Hematogenous Route (Bloodstream)}

Coronaviruses can also travel through the bloodstream, where it infects the endothelial cells of the blood-brain-barrier (BBB) or takes control of peripheral blood cells (mostly monocytes/macrophages), which are infected in the early stages in the airways. CoVs can infiltrate the brain through the release of inflammatory mediators that cause meningeal inflammation and damage to the BBB (Tohidpour et al., 2017). A study used transmission electron microscopy and found SARS-CoV2 in frontal lobe tissue, suggesting that the virus most likely enters the brain through the hematogenous route (Paniz-Monodolfi et al., 2020). 


\section{Lymphatic Route}

SARS-CoV can directly invade hilar lymph nodes and mesenteric root lymph nodes both in humans and civets, especially in the lungs and intestine ( $\mathrm{Li}$ et al., 2020). While the brain was previously believed to not have any lymphatic vessels, later findings demonstrated that lymphatic drainage exists in the brain (Louveau et al., 2015). This glymphatic pathway contains olfactory/cervical lymphatic vessels, which could allow the virus to directly infiltrate the brain (Bostancıklığlu et al., 2020).

\section{Symptoms}

\section{Neural}

COVID-19, especially in severe forms, causes neurological symptoms such as impaired consciousness, skeletal muscle injury, and acute cerebrovascular disease, according to a study performed in China (Kwong et al., 2020). Researchers suggested a potential involvement of the central nervous system (CNS) and peripheral nervous system (PNS); other possible PNS symptoms included hypogeusia (loss of or reduction in taste) and hyposmia (loss of or reduction in smell and odor detection). 13\% of patients with COVID-19 later had acute cerebrovascular disease - including ischaemic stroke, cerebral venous sinus thrombosis, and cerebral hemorrhage (Lahiri et al., 2020).

Of patients in a study done in France who had acute respiratory distress syndrome, $84 \%$ experienced neurological symptoms. There was evidence of encephalopathy corticospinal tract dysfunction, agitation, and delirium. A small group of patients was also scanned with magnetic resonance imaging (MRI) and perfusion brain imaging, which demonstrated leptomeningeal enhancement and bilateral frontotemporal hypoperfusion. Two patients also showed evidence for a small acute ischaemic stroke (Kwong et al., 2020). These trials demonstrate a shared prevalence of stroke as a result of infection in addition to a wide variety of other detrimental impacts on the brain and spinal cord.

\section{Psychological}

It was correspondingly identified that the environment surrounding patients with COVID can trigger multiple psychological responses, including anxiety, suicidal ideation, agitation, suspiciousness, auditory hallucinations, and decreased sleep.

Testing from a case study in New York showed elevated levels of C-reactive protein (CRP) in patients with increased psychotic symptoms. CRP is a known marker of inflammatory response, and its presence was postulated to trigger schizophreniform psychosis (Ferrando et al., 2020). The increased number of cytokines caused by the cytokine storm, especially proinflammatory ones such as IL-6, can induce inflammation that causes neuropsychiatric symptoms. Neuroinflammation is known to be a factor in a large number of neurological and psychiatric diseases. It has been connected to early stages of Alzheimer's, Parkinson's, and multiple sclerosis (MS), as well as changes in hypothalamic pituitary adrenal (HPA) axis regulation and neurotransmitter metabolism. The structural changes occurring due to neuroinflammation can lead to other potential cognitive and behavioral deficits. However, the inflammatory reaction itself is not the only source that can cause psychosis. The increased use of corticosteroids to treat the inflammatory reactions has also been linked to psychosis, meaning both the response and its solution have the potential to cause harmful effects to the patient. Although the amount of data on COVID-19 is limited due to the recent nature of the virus, scientists have analyzed past coronaviruses such as SARS Cov-1 to help predict the possible long-term neurological effects. These past diseases have been known to have effects such as anxiety, depression, suicidal ideation, mania, and hallucinosis. As more people are infected with COVID-19, it is imperative to assess these patients in order to rule out untreated long-term psychiatric effects. The population with the greatest risk of adverse effects from the inflammation is the elderly. Neurodegenerative age-related diseases can be sped up by the inflammatory 
environment, so extra care should be taken with controlling the inflammation in older populations of patients. Although the effects on children and adolescents is not immediately evident, there is still the potential for long-term effects due to synaptic pruning during their brains' growth stages (Serrano-Castro et al., 2020).

From the psychological perspective, a cross-sectional survey done in China revealed that COVID-19 also had a severe impact on the patient's mental health. In an anonymous online survey using a technique where respondents recruited additional subjects, or the "snowball sampling strategy", researchers collected their data from 1210 patients in China in early 2020. (Wang et al., 2020).

The survey revealed that $53.8 \%$ of respondents saw the psychological impact of the outbreak as moderate or severe, $16.5 \%$ reported moderate to severe depressive symptoms, $28.8 \%$ reported moderate to severe anxiety symptoms, and 8.1\% reported moderate to severe stress levels (Wang et al., 2020). Most respondents spent 20-24 h per day at home, were worried about close ones contracting COVID-19, and were satisfied with the available health information (Wang et al., 2020). Female gender, student status, symptoms such as dizziness, and poor self-rated health status were strongly associated with a greater psychological impact of the outbreak, including higher levels of stress, anxiety, and depression (Wang et al., 2020).

\title{
COVID-19's Indirect Effects on Healthcare Workers and the General Population
}

\author{
Impacts of Isolation and Paranoia on the General Population
}

There are significant merits of social distancing concerning the spread of COVID-19, but this can contribute to feelings of isolation, loneliness, stress, and fear-all of which can be triggers that contribute to the worsening of mental illness (McIntyre, 2020). Among those diagnosed with generalized anxiety disorder, many may fear catching or dying from COVID-19, which increases anxiety levels. Obsessive compulsive disorder (OCD) is an anxiety disorder that occurs when a person gets stuck in a recurring cycle of obsessions and compulsions. For those diagnosed with OCD, specifically those whose obsessions and compulsions revolve around cleanliness and germs, a global pandemic may exacerbate symptoms. Similar to anxiety, for those with depression, fear and isolation can be very dangerous. Without outside consultation, people may develop a sense of hopelessness or maybe paralyzed by fear, leading them to neglect both their health and wellbeing. There are many other existing mental illnesses that are, although not connected with the virus, continue to affect the mental health and the lives of those who have it. Thus, the heightened fear and anxiety, trauma, and stress due to the threat of the pandemic have severe consequences on mental health.

\section{The Impacts of Loneliness}

Social distancing is one of the many ways that is being suggested to help diminish the exponential growth of hospitalized Coronavirus patients. It is recommended for everyone to stay inside of their homes unless it is necessary to go out. The impact of quarantine has also increased the number of people feeling lonelier during self-isolation. The stresses caused by the contrasting social environment of isolation that both the youth and adults do not experience in their normal day at school or in public spaces, respectively, have made a great impact on the number of prescriptions filled per week. For instance, antidepressant, anti-anxiety, and anti-insomnia medications increased $21 \%$ between February 16 and March 15 (Cigna, 2020). In fact, the sales of these medications peaked the week of March 15, which was when COVID-19 was announced a pandemic (Cigna, 2020). Though the restrictions on gathering within some states have been lifted for a while, the United States' cases of Coronavirus have increased, affecting the population's overall mental health.

\section{Changes in Sleep Patterns among the General Population}

While one might imagine that the inability to go to school and engage in other outdoor activities has positively increased the amount of sleep individuals are able to get, COVID-19 has actually shown to create adverse effects on 
sleeping habits of those participating in the lockdown. Although many indeed get far more hours of sleep than usual, that is not always beneficial. This causes a huge disturbance in regular sleep cycles and results in more lethargic feelings throughout the day, and the stress of doing work outside of a normal routine and a lack of physical movement can further damage one's sleep quality.

A sleep study conducted in Austria, Germany, and Switzerland testing people's sleep quality before and after the COVID-19 lockdown revealed that overall sleep quality and duration slightly decreased as a result of the virus, and mental health seemed to be negatively affected as well (Blume et al., 2020). This is harmful to the general population, as sleep is essential to proper physical and mental health. Sleep deprivation has been shown to negatively impact a part of the brain known as the hippocampus, which is imperative for learning and memory. After not getting a sufficient amount of sleep, individuals are more likely to have a difficult time remembering and retaining information. Lack of sleep also impairs functioning of the amygdala, a part of the brain responsible for processing emotions. Sleep is also important for physical growth and other bodily processes, making sleep deprivation detrimental for those already suffering physical illnesses and conditions (e.g., coronavirus).

It is important to recognize the importance of sleep and its benefits to individuals, while also emphasizing the effects COVID-19 has on it. During the lockdown, people must take special care in getting the proper amount of sleep, as well as decreasing stress, pressure, and anything else that could impair one's sleep quality.

Moreover, the current COVID-19 pandemic is causing increased rates of stress and anxiety. In regards to the general public, the fear of spreading the virus carries consequences. Risks of contracting the virus and worries over passing it on to high-risk loved ones were recorded to build up, especially in essential workers (Tsamakis et al., 2020). With an increase in stress and anxiety, observing an increase in insomnia and other illnesses related seems inevitable.

In addition to health stressors, we are troubled by social and financial stressors (Devanathan, 2020). Whether it is about the health aspect of our current crisis or financial, social, or economic state of our world due to the pandemic, we are inevitably affected by global events that alter our ability to sleep at night. The resulting lack of sleep that many are experiencing results in a weakened immune system and a higher risk of contracting the virus or more difficulty overcoming the virus.

\section{Paranoia}

Furthermore, studies have shown that patients who were tested positive for COVID-19 could undergo mental anxiety even without the presence of observable symptoms (Ferrando et al., 2020). In a case report observed by the Abbott Laboratories in Chicago, a 30-year-old male without any respiratory or gastrointestinal symptoms of COVID-19 was brought to ED for his altered mental condition. Though he was not observed to have any outward symptoms of the virus, he tested positive for COVID-19 (Ferrando et al., 2020).

In another case report, a 34-year-old female reported a similar set of symptoms as the previously identified case, where she suffered anxiety and hallucination of a "fire burning up inside." While she had a history of a significant panic disorder, she also did not show any respiratory or gastrointestinal symptoms, anosmia, or ageusia. Nevertheless, she also tested positive for COVID-19 in the ED (Ferrando et al., 2020).

In both cases, the patients never showed any COVID-19 related respiratory or gastrointestinal symptoms. Yet both patients expressed their experience of auditory hallucinations and anxiety/stress, which were not COVID-19 related concerns. The fact that their stress did not originate from preoccupation towards COVID-19 and the increased inflammation markers in CRP indicates a virus-associated inflammatory trigger. Such newly reported cases of psychosis imply that the psychological symptoms expressed in both cases may have been derived from the extreme stress towards the recent virus pandemic, especially when both patients were mentally vulnerable. It is also worthy to note that these patients experienced abrupt delirium and sudden-onset psychosis (Ferrando et al., 2020).

\section{Social and Cultural Impacts on Reaction to Social Guidelines}

Another important aspect of the pandemic is the observation of the universal as well as unique psychologies encompassed by different people that influence how they react to the pandemic in terms of threat and social guidelines. 
Most people are influenced by social contexts and perception of fear and threat in similar matters. For instance, a meta-analysis revealed that people are less likely to change behavior when they feel that they are not capable of dealing with the threat (Strunk et al., 2005). However, it is also known that negative framing of the pandemic sensitizes people to neglected risk for themselves or others (Bavel et al., 2020). Additionally, many people experience optimism bias, in which they have a false sense of comfort of bad things falling upon themselves. Thus, due to the large influence of social contexts in an individual's reaction to a large threat like the pandemic, social measures can play a large role in fostering unity and control. For instance, coordinated efforts among individuals, communities, and governments promote feelings of cooperation and shared values, which are vital in such turbulent times (Bavel et al., 2020). However, leaders must be careful to share positive reinforcement and to emphasize notions that they are working for the overall greater good of the community, as threats often lead to distrust and adverse reactions to the suggested guidelines (Bavel et al., 2020). Additionally, people are likely to follow the choices made by trusted individuals and thus wide community efforts are essential in promoting healthy habits in order to adhere to the appropriate social guidelines (Bavel et al., 2020). In fact, influential figures such as leaders and celebrities act as role models that can motivate people to act with kindness and selflessness (Schnall et al., 2011, Yang et al., 2018). As people tend to underestimate healthy behaviors while overestimating unhealthy behaviors, positive habits must be promoted through accurate information and social measures such as appropriate behavior in local communities (Bavel et al., 2020). Political polarization also presents problems in the forms of "Attitudinal polarization," which consists of taking extreme positions on issues, and "Affective Polarization," which consists of individuals distrusting and strongly opposing the conflicting views of the other parties (Westwood et al., 2018, Iyengar et al., 2019). As a result, the community is often broken up, which has been proven to be detrimental in promoting wide, communal efforts enforcing healthy social behaviors. People are also likely to seek and be influenced by biased, potentially inaccurate representations of the situation. Furthermore, people are psychologically influenced to attach large causes to large events, further developing conspiracy theories that may proliferate inaccurate information (Leman et al., 2007). In order to combat this, politicians must be able to take bipartisan stances towards the appropriate measures, and accurate information needs to be distributed.

However, in addition to these universal effects on psychology, there are distinct cultural trends that differentiate how people react to crises like the COVID-19 pandemic. For instance, western cultures (North America and Europe) tend to emphasize values of independence, as opposed to a greater emphasis on the overall communal good over personal desires in Asian countries (Kitayama et al., 2018). Additionally, western cultures often incorporate greater instances of physical intimacy that may present habits prone to spread of the virus (Kraus et al., 2019).

\section{Socioeconomic Impacts of Pandemic on Child Neural Development}

The unprecedented times of the COVID-19 pandemic have also incited severe effects on the economy. In fact, the global extreme poverty rate is expected to increase from $8.23 \%$ in 2019 to $8.82 \%$ under a baseline scenario (The World Bank, 2020) or $9.18 \%$ under a downside scenario, which encapsulates the first global extreme poverty since 1998 and undermining progress made since 2017. Besides, millions of families are facing unemployment and closed businesses, leading to financial insecurities. Thus, it is important to recognize the potential long-term psychological and neural effects on affected children, as they are in the prime, sensitive age of development.

Children who grow up in poverty often live in adverse environments consisting of racial discrimination, violence, housing instability, and harsh parenting as a result of harsh discipline since some parents underwent poverty, are mentally unstable, or do not have resources to seek help. This disrupts emotional, cognitive, behavioral, and physical health and development, particularly in the areas of the brain related to emotion regulation and cognition. Additionally, these adverse effects may lead to severe conditions such as heart disease, substance abuse, and depression (Braveman, 2009, Shonkoff et al., 2012). However, these effects can be offset by stable, safe, and supportive home environments by reinforcing qualities of routine and predictability and trust in relationships. Unfortunately, some parents raising their children in these conditions were born in poverty themselves and may suffer from traumas and 
unconfidence in their ability to provide an ideal environment for development. Though there are multiple resources for child behavioral health screening and a great amount of research on the effectiveness of parent-focused methods of combating adverse developmental environments, interests in implementing parenting programs in primary care and educating primary care providers about parenting strategies are a more novel area of growth (Francis et al., 2018). It is even more important during these times to implement screening methods for parent and child behavior and to proliferate healthy parenting strategies.

\section{Impacts of Pressures on Health Care Workers}

The prevalence of COVID-19's detrimental effects can be observed beyond the direct impact of the disease on the patient, specifically when considering the toll it takes on the mental wellbeing of healthcare professionals on the frontlines of the pandemic. Healthcare workers in Greece specifically have reported an increased sense of anxiety, uncertainty, vulnerability, and overall distress in the past with the SARS outbreak (Tsamakis et al., 2020). These feelings are very likely to also be present in the COVID-19 pandemic, with healthcare workers facing challenges similar to those in previous major outbreaks, such as a considerable increase in patients. In fact, these feelings are most likely heightened since COVID-19 has presented to be a larger outbreak than SARS, meaning that healthcare workers are dealing with an increased number of patients and thus, greater risks of being infected. Other difficulties that were exacerbated by the COVID-19 pandemic include a high-pressure atmosphere. Some factors contributing to this strained environment result from the public criticism, lack of sufficient resources, necessary speed and urgency in decision making and treatment coupled with potential adverse effects, and danger associated with increased exposure to the virus, among others (Tsamakis et al., 2020). Increased dangers of infection and harsh work conditions foster a source of potentially unbearable stress. All of these extra stressors pile on top of an already demanding career, feelings of helplessness, and uncooperative patients (Tsamakis et al., 2020).

However, one aspect to note is that in some cases, these barriers have not altered the frontline medical workers' motivation to continue their necessary service, similar to their dedication demonstrated in past pandemics. In fact, there has been an observed increase in levels of commitment and resilience in treating patients among the workers in the Greece National Health Service (Tsamakis et al., 2020).

Nevertheless, the physical and mental strain created by the conditions of working such taxing shifts with extensive protective equipment and few breaks has negative psychological consequences. According to a study performed in China, anxiety, depression, and stress disorders were frequent among these healthcare workers due to a feeling of loss of control and general uncertainty (Tsamakis et al., 2020). Similarly, an online nationwide survey performed with over 2,000 participants over the age of 16 from China analyzed factors contributing to mental health issues, including but not limited to anxiety and depression (Zhang et al., 2020). Assessed using the Patient Health Questionnaire - 4, the Symptom Checklist - 90 - revised, and similar scales, the study approximately indicated a higher prevalence in rates of insomnia (38.4\% vs. 30.5\%1), anxiety (13.0\% vs. 8.5\%), depression (12.2\% vs. 9.5\%), somatization ( $1.6 \%$ vs. $0.4 \%$ ), and obsessive-compulsive symptoms (5.3\% vs. $2.2 \%)$. Among the medical workers, a few variables encompassed in the analysis were gender, location, risk of COVID-19 contact, and medical history, all of which presented risk factors for developing mental health issues. Additionally, a study observing healthcare professionals in a hospital coping with COVID-19 in China revealed a high rate of anxiety and stress disorders among frontline medical staff, especially among nurses (Huan et al., 2020).

Overall, the augmentation of risk factors and prevalence rates suggests a risk of allostatic overload and other destructive psychological effects on healthcare workers, highlighting the necessity of workplace practices to minimize these burdens. The increase in these mental health disorders suggests that a lack of prevention, a long-term workload, a high risk of exposure to patients with COVID-19, and the shortage of medical protective equipment are all stress factors that have been contributing to the deteriorating mental health of healthcare professionals. With an increase in education and knowledge regarding treatment of COVID-19, the psychological effects on frontline workers may be reduced. Moreover, proper procedures addressing mental health concerns among these workers must be enacted. To 
combat COVID-19's detrimental impact on healthcare workers' psychosocial wellbeing, it has been recommended that healthcare workers should be provided with sufficient medical equipment, adequate working conditions, rest, and recovery programs. Additionally, story sharing among healthcare workers was highlighted as being an important aspect of recovery, as it can serve to reinforce positive outcomes and traits in workers (Zhang et al., 2020).

\section{Conclusion}

The virus presents observable neurological effects, causing problems ranging from dizziness, headaches, nausea, and vomiting to brain tissue edema and partial neuronal degeneration. COVID-19 can directly attack the nervous system entering through methods such as through the olfactory bulbs and through an inflammatory response. There is thus a need to develop a variety of treatments accounting for these multiple sources and the variety of symptoms that may emerge. COVID-19 has also seen a rise in new-onset psychiatric conditions such as hallucinations and delusions, which have been tied to the presence of the inflammatory protein CRP. This raises questions about the long-term psychological impacts of the virus and the connections between the routes of entry and symptoms.

Additionally, COVID-19 has indirectly impacted mental wellbeing in the public through social distancing, leading to a dramatic increase in feelings of isolation, loneliness, stress, and fear, which may trigger serious conditions such as depression. Families of patients and healthcare workers further experience significant levels of anxiety and depression due to work overload and fear about the spread of the virus. The general population should make sure to stay in touch with family and friends to combat loneliness, and hospitals should adopt measures for psychological support and interventions to protect healthcare workers. These measures could be in the form of more balanced work schedules and in the form of greater incentives. Furthermore, there is a need for more widespread mental health services for the general population.The virus is also expected to cause an increase in poverty around the world, which would further contribute to long term psychological effects on children exposed to racial discrimination, violence, housing instability, and harsh parenting. These can disrupt emotional, cognitive, behavioral, and physical health and development in children, highlighting a need for services that can monitor and provide counseling in these situations, such as the parent-focused interventions and primary care initiatives mentioned.

However, the psychological impact differs based on factors such as cultural values and leadership. Though there are universal influences, such as the panic-inducing use of negative framing, differing values on social responsibility and organized, strong leadership change how the public responds. This showcases the importance of unity and hope fostered by leadership and highlights unique barriers that certain countries, such as those that engage in more physical contact, may need to account for.

Since this virus is fairly new and very little is known about it and its future effects, more research needs to be done to fully understand its neural and psychological effects. In addition to exploring neural and psychological symptoms of the virus, there is a need to monitor the long-term effects of the virus, whether it be directly in patients or indirectly in health care workers and the general population.

\section{Acknowledgments}

We would like to thank Dr. Paul Mathews and Dr. Matt Wright from the Lundquist Institute for their continuous guidance and advice. We are extremely appreciative of all the valuable mentorship they gave us in completing this literature review. We would also like to express our gratitude to the Lundquist Institute team for connecting us with Dr. Mathews and Dr. Wright and their support throughout the process. 


\section{References}

America's State of Mind: Use of Mental Health Medications Increasing with Spread of Coronavirus. (2020, April 16). Retrieved from https://www.cigna.com/newsroom/news-releases/2020/americas-state-of-mind-use-ofmental-health-medications-increasing-with-spread-of-coronavirus

Bavel, J., Baicker, K., Boggio, P., Capraro, V., Cichocka, A., Cikara, M., . . Willer, R. (2020, April 30). Using social and behavioural science to support COVID-19 pandemic response. Retrieved from https://www.nature.com/articles/s41562-020-0884-z

Bostanciklığlu, M. (2020, July). SARS-CoV2 entry and spread in the lymphatic drainage system of the brain. Retrieved from https://www.ncbi.nlm.nih.gov/pmc/articles/PMC7189839/

Blume, C., Schmidt, M. H., \&amp; Cajochen, C. (2020, June 10). Effects of the COVID-19 lockdown on human sleep and rest-activity rhythms. Retrieved August 03, 2020, from https://www.ncbi.nlm.nih.gov/pmc/articles/PMC7284244/

Bohmwald, K., Gálvez, N., Ríos, M., \& Kalergis, A. (2018, October 26). Neurologic Alterations Due to Respiratory Virus Infections. Retrieved from https://www.ncbi.nlm.nih.gov/pmc/articles/PMC6212673/

Braveman, P., \& Barclay, C. (2009, November 01). Health Disparities Beginning in Childhood: A Life-Course Perspective. Retrieved from https://pediatrics.aappublications.org/content/124/Supplement_3/S163

Burki, T. (2020, August). The indirect impact of COVID-19 on women. Retrieved August 03, 2020, from https://www.thelancet.com/journals/laninf/article/PIIS1473-3099(20)30568-5/fulltext

Chen, C., Lin, K., Chen, C., Wong, A., \& Huang, J. (1970, January 01). Central nervous system manifestations of neonatal lupus: A systematic review. Retrieved August 03, 2020, from https://www.mendeley.com/catalogue/dc616840-e18c-3707-99bd-f15f80cbb37d/

Chiu, A., Fischbein, N., Wintermark, M., Zaharchuk, G., Yun, P. T., \& Zeineh, M. (2020, September 15). COVID19-induced anosmia associated with olfactory bulb atrophy. Retrieved from https://link.springer.com/article/10.1007/s00234-020-02554-1

Cho, R., To, Z., Yeung, Z., Tso, E., Fung, K., Chau, S., . . Ku, P. (2020, August 13). COVID-19 Viral Load in the Severity of and Recovery From Olfactory and Gustatory Dysfunction. Retrieved from https://onlinelibrary.wiley.com/doi/10.1002/lary.29056

Chung, M., Choi, W., Jeong, H., Lee, J., \& Kim, J. (2018, March 01). MR Imaging-Based Evaluations of Olfactory Bulb Atrophy in Patients with Olfactory Dysfunction. Retrieved from http://www.ajnr.org/content/39/3/532

Devanathan, S. (2020, April 21). The connection between coronavirus and sleep issues. Retrieved August 03, 2020, from https://www.parkview.com/community/dashboard/the-connection-between-coronavirus-and-sleepissues

Ferrando, S., Klepacz, L., Lynch, S., Tavakkoli, M., Dornbush, R., Baharani, R., . . Bartell, A. (2020, May 19). COVID-19 Psychosis: A Potential New Neuropsychiatric Condition Triggered by Novel Coronavirus 
Infection and the Inflammatory Response? Retrieved August 03, 2020, from https://www.ncbi.nlm.nih.gov/pmc/articles/PMC7236749/

Francis, L., DePriest, K., Wilson, M., \& Gross, D. (2018, September 30). Child Poverty, Toxic Stress, and Social Determinants of Health: Screening and Care Coordination. Retrieved from https://www.ncbi.nlm.nih.gov/pmc/articles/PMC6699621/

Giacomelli, A., Pezzati, L., Conti, F., Bernacchia, D., Siano, M., Oreni, L., . . Galli, M. (2020, March 26). Selfreported Olfactory and Taste Disorders in Patients With Severe Acute Respiratory Coronavirus 2 Infection: A Cross-sectional Study. Retrieved from https://www.ncbi.nlm.nih.gov/pmc/articles/PMC7184514/

Gu, J., Gong, E., Zhang, B., Zheng, J., Gao, Z., Zhong, Y., . . Leong, A. (2005, August 1). Multiple organ infection and the pathogenesis of SARS. Retrieved from https://www.ncbi.nlm.nih.gov/pmc/articles/PMC2213088/

Hopkins, C., Surda, P., \& Kumar, N. (2020, June 01). Presentation of new onset anosmia during the COVID-19 pandemic. Retrieved from https://pubmed.ncbi.nlm.nih.gov/32277751/

Huan, J., Han, M., Luo, T., Ren, A., \&amp; Zhou, X. (2020, March 20). [Mental health survey of medical staff in a tertiary infectious disease hospital for COVID-19]. Retrieved August 03, 2020, from https://pubmed.ncbi.nlm.nih.gov/32131151/

Iyengar, S., Lelkes, Y., Levendusky, M., Malhotra, N., \& Westwood, S. (2018, December 10). The Origins and Consequences of Affective Polarization in the United States. Retrieved from https://www.annualreviews.org/doi/10.1146/annurev-polisci-051117-073034

Kim, J., Heo, J., Kim, H., Song, S., Park, S., Park, T., . . Choi, J. (2017, June 30). Neurological Complications during Treatment of Middle East Respiratory Syndrome. Retrieved from https://thejen.com/DOIx.php?id=10.3988\%2Fjen.2017.13.3.227

Kitayama, S., Park, J., Miyamoto, Y., Date, H., Boylan, J., Markus, H., . . Ryff, C. (2018, January 30). Behavioral Adjustment Moderates the Link Between Neuroticism and Biological Health Risk: A U.S.-Japan Comparison Study. Retrieved from https://www.ncbi.nlm.nih.gov/pmc/articles/PMC5940540/

Kraus, B., \& Kitayama, S. (2019, July 25). Interdependent self-construal predicts emotion suppression in Asian Americans: An electro-cortical investigation. Retrieved from https://www.sciencedirect.com/science/article/abs/pii/S0301051118304782?via=ihub

Kwong, K., Mehta, P., Shukla, G., \& Mehta, A. (1970, January 01). COVID-19, SARS and MERS: A neurological perspective. Retrieved August 03, 2020, from https://www.mendeley.com/catalogue/3669107a-7ad5-32ca$\underline{\mathrm{a} 822-8 \mathrm{c} 0 \mathrm{~d} 8143 \mathrm{~b} 40 \mathrm{c} /}$

Lahiri D, Ardila A (April 29, 2020) COVID-19 Pandemic: A Neurological Perspective. Cureus 12(4): e7889. DOI 10.7759/cureus.7889. Retrieved August 03, 2020 from https://assets.cureus.com/uploads/review article/pdf/30897/1592535731-20200619-18809-tp59t.pdf

Leman, P., \& Cinnirella, M. (2007, January). A major event has a major cause: Evidence for the role of heuristics in reasoning about conspiracy theories. Retrieved from 
https:/www.researchgate.net/profile/Patrick_Leman/publication/285852159_A_major_event_has_a_major_cause_Evidence_for_the_role_of_heuristics_in_reasoning_about_conspiracy_theories/links/5de0d2b4a6fdcc2837f3ee98/A-major-event-has-a-major-cause-Evidence-for-the-role-of-heuristics-in-reasoning-about-conspiracy-theories.pdf

Li, Y., Fu, L., Gonzales, D., \& Lavi, E. (2004, April). Coronavirus neurovirulence correlates with the ability of the virus to induce proinflammatory cytokine signals from astrocytes and microglia. Retrieved from https://www.ncbi.nlm.nih.gov/pmc/articles/PMC371061/

Li, Z., Liu, T., Yang, N., Han, D., Mi, X., Li, Y., . . Guo, X. (1970, January 01). Neurological manifestations of patients with COVID-19: Potential routes of SARS-CoV-2 neuroinvasion from the periphery to the brain. Retrieved August 03, 2020, from https://www.mendeley.com/catalogue/57f01658-82ad-384d-bc149b6540582090/

Louveau, A., Smirnov, I., Keyes, T., Eccles, J., Rouhani, S., Peske, J., . . Kipnis, J. (2015, July 16). Structural and functional features of central nervous system lymphatic vessels. Retrieved from https://www.ncbi.nlm.nih.gov/pmc/articles/PMC4506234/

Lu, R., Zhao, X., Li, J., Niu, P., Yang, B., Wu, H., Wang, W., Song, H., Huang, B., Zhu, N., Bi, Y., Ma, X., Zhan, F., Wang, L., Hu, T., Zhou, H., Hu, Z., Zhou, W., Zhao, L., Chen, J., ... Tan, W. (2020). Genomic characterisation and epidemiology of 2019 novel coronavirus: implications for virus origins and receptor binding. Lancet (London, England), 395(10224), 565-574. https://doi.org/10.1016/S0140-6736(20)30251-8

Mao, L., Jin, H., Wang, M., Hu, Y., Chen, S., He, Q., . . Hu, B. (2020, April 10). Neurologic Manifestations of Hospitalized Patients With Coronavirus Disease 2019 in Wuhan, China. Retrieved from https://jamanetwork.com/journals/jamaneurology/fullarticle/2764549

McIntyre, M., \&amp; Fazel, F. (2020, April 24). Coronavirus and Mental Health: How COVID-19 is Affecting Millions of Americans. Retrieved August 03, 2020, from https:/www.psycom.net/coronavirus-mental-health

Mental Health \&amp; COVID-19: What Parents and Caregivers Should Know. (2020, May 5). Retrieved August 03, 2020, from https://www.bgca.org/news-stories/2020/May/Mental-Health-COVID-19-What-Parents-andCaregivers-Should-Know

Mental Health and Coping During COVID-19. (2020, July 1). Retrieved August 03, 2020, from https:/www.cdc.gov/coronavirus/2019-ncov/daily-life-coping/managing-stress -anxiety.html

Older Adults Loneliness Fact Sheet. (2018, September 11). Retrieved August 03, 2020, from https://www.artandhealing.org/older-adults-loneliness-fact-sheet/?gclid=CjOKCQjwgo 5BRDuARIsADDEntSndSirdvcDAuevsuU oHlcJrm2fU0o9lqy 3D1VYqe1nDbFZw C0aAiWGEALw wcB

Paniz-Mondolfi, A., Bryce, C., Grimes, Z., Gordon, R., Reidy, J., Lednicky, J., . . Fowkes, M. (2020, July). Central nervous system involvement by severe acute respiratory syndrome coronavirus-2 (SARS-CoV-2). Retrieved from https://www.ncbi.nlm.nih.gov/pmc/articles/PMC7264598/

Projected poverty impacts of COVID-19 (coronavirus). (n.d.). Retrieved from https://www.worldbank.org/en/topic/poverty/brief/projected-poverty-impacts-of-COVID-19 
Saad, M., Omrani, A., Baig, K., Bahloul, A., Elzein, F., Matin, M., . . Albarrak, A. (2014, December). Clinical aspects and outcomes of 70 patients with Middle East respiratory syndrome coronavirus infection: A singlecenter experience in Saudi Arabia. Retrieved from https://www.ncbi.nlm.nih.gov/pmc/articles/PMC7110769/

Schnall, S., \& Roper, J. (2011, October 05). Elevation Puts Moral Values Into Action - Simone Schnall, Jean Roper, 2012. Retrieved from https://journals.sagepub.com/doi/10.1177/1948550611423595

Serrano-Castro, P., Estivill-Torrús, G., Cabezudo-García, P., Reyes-Bueno, J., Petersen, N., Aguilar-Castillo, M., . . . Fonseca, F. (2020, May 08). Impact of SARS-CoV-2 infection on neurodegenerative and neuropsychiatric diseases: A delayed pandemic? Retrieved August 03, 2020, from https://www.sciencedirect.com/science/article/pii/S2173580820300572

Shonkoff, J., Garner, A., The Committee On Psychosocial Aspects Of Child And Family Health, C., Siegel, B., Dobbins, M., Earls, M., . . Wood, D. (2012, January 01). The Lifelong Effects of Early Childhood Adversity and Toxic Stress. Retrieved from https://pediatrics.aappublications.org/content/129/1/e232

Strunk, D., Lopez, H., \& DeRubeis, R. (2005, August 25). Depressive symptoms are associated with unrealistic negative predictions of future life events. Retrieved from https:/pubmed.ncbi.nlm.nih.gov/16126162/

Tohidpour, A., Morgun, A., Boitsova, E., Malinovskaya, N., Martynova, G., Khilazheva, E., .. . Salmina, A. (2017, June 20). Neuroinflammation and Infection: Molecular Mechanisms Associated with Dysfunction of Neurovascular Unit. Retrieved from https://www.ncbi.nlm.nih.gov/pmc/articles/PMC5476750/

Tsai, L., Hsieh, S., \& Chang, Y. (2005, September). Neurological manifestations in severe acute respiratory syndrome. Retrieved from https://pubmed.ncbi.nlm.nih.gov/16252612/

Tsamakis, K., Rizos, E., Manolis, A., Chaidou, S., Kympouropoulos, S., Spartalis, E., . . Triantafyllis, A. (2020, June). COVID-19 pandemic and its impact on mental health of healthcare professionals. Retrieved August 03, 2020, from https://www.ncbi.nlm.nih.gov/pmc/articles/PMC7185082/

Wan, S., Yi, Q., Fan, S., Lv, J., Zhang, X., Guo, L., . . Chen, Y. (2020, February 12). Characteristics of lymphocyte subsets and cytokines in peripheral blood of 123 hospitalized patients with 2019 novel coronavirus pneumonia (NCP). Retrieved from https://www.medrxiv.org/content/10.1101/2020.02.10.20021832v1

Wang, C., Pan, R., Wan, X., Tan, Y., Xu, L., Ho, C., \& Ho, R. (1970, January 01). Immediate psychological responses and associated factors during the initial stage of the 2019 coronavirus disease (COVID-19) epidemic among the general population in China. Retrieved August 03, 2020, from https://www.mendeley.com/catalogue/a7171a9b-2e46-3775-8e42-7b4bd00eae70/

Wang, H., Li, X., Li, T., Zhang, S., Wang, L., Wu, X., \& Liu, J. (2020, April 24). The genetic sequence, origin, and diagnosis of SARS-CoV-2. Retrieved from https://www.ncbi.nlm.nih.gov/pmc/articles/PMC7180649/

Westwood, S., Iyengar, S., Walgrave, S., Leonisio, R., Miller, L., \& Strijbis, O. (2017, August 11). The tie that divides: Cross-national evidence of the primacy of partyism. Retrieved from https://ejpr.onlinelibrary.wiley.com/doi/abs/10.1111/1475-6765.12228 
Wu, Y., Xu, X., Chen, Z., Duan, J., Hashimoto, K., Yang, L., . . Yang, C. (2020, March 30). Nervous system involvement after infection with COVID-19 and other coronaviruses. Retrieved August 03, 2020, from https://www.sciencedirect.com/science/article/pii/S0889159120303573?via=ihub

Xiang P., Xu X.M., Gao L.L., Wang H.Z., Xiong H.F., Li R.H. First case of 2019 novel coronavirus disease with Encephalitis. ChinaXiv. 2020;T202003:00015.

Yang, X., Pavarini, G., Schnall, S., \& Immordino-Yang, M. (2018, November 8). Looking up to virtue: Averting gaze facilitates moral construals via posteromedial activations. Retrieved from https://pubmed.ncbi.nlm.nih.gov/30212913/

Zhang, Q., Ding, Y., Hou, J., He, L., Huang, Z., Wang, H., . . Lu, Y. (2003, November). [Detection of severe acute respiratory syndrome (SARS)-associated coronavirus RNA in autopsy tissues with in situ hybridization]. Retrieved from https://pubmed.ncbi.nlm.nih.gov/14625166/

Zhang, W., Wang, K., Yin, L., Zhao, W., Xue, Q., Peng, M., .. Wang, H. (2020, April 9). Mental Health and Psychosocial Problems of Medical Health Workers during the COVID-19 Epidemic in China. Retrieved August 03, 2020, from https://pubmed.ncbi.nlm.nih.gov/32272480/ 